\title{
Video Article \\ Visualization and Quantification of Mesenchymal Cell Adipogenic Differentiation Potential with a Lineage Specific Marker
} \author{
Pritika Narayan $^{1,6}$, P. Rod Dunbar ${ }^{1,7}$ \\ ${ }^{1}$ School of Biological Sciences, The University of Auckland \\ ${ }^{2}$ Research School of Biology, The Australian National University \\ ${ }^{3}$ Auckland Cancer Society Research Centre, Faculty of Medical and Health Sciences, The University of Auckland \\ ${ }^{4}$ Department of Plastic Surgery, Counties Manukau District Health Board \\ ${ }^{5}$ Department of Surgery, Faculty of Medical and Health Sciences, The University of Auckland \\ ${ }^{6}$ Biomedical Imaging Research Unit, Faculty of Medical and Health Sciences, The University of Auckland \\ ${ }^{7}$ Maurice Wilkins Centre, The University of Auckland
}

Jennifer Eom ${ }^{1}$, Vaughan Feisst ${ }^{1}$, Louis Ranjard ${ }^{2}$, Kerry Loomes $^{1}$, Tanvi Damani ${ }^{1}$, Victoria Jackson-Patel ${ }^{1,3}$, Michelle Locke ${ }^{4,5}$, Hilary Sheppard ${ }^{1}$,

Correspondence to: Pritika Narayan at p.narayan@auckland.ac.nz

URL: https://www.jove.com/video/57153

DOI: doi:10.3791/57153

Keywords: Biology, Issue 133, Adipose derived stromal cells, differentiation, adipogenesis, imaging, immunocytochemistry, immunofluorescence, quantification, high content screening

Date Published: 3/31/2018

Citation: Eom, J., Feisst, V., Ranjard, L., Loomes, K., Damani, T., Jackson-Patel, V., Locke, M., Sheppard, H., Narayan, P.,

Dunbar, P.R. Visualization and Quantification of Mesenchymal Cell Adipogenic Differentiation Potential with a Lineage Specific Marker. J. Vis. Exp.

(133), e57153, doi:10.3791/57153 (2018).

\section{Abstract}

Several dyes are currently available for use in detecting differentiation of mesenchymal cells into adipocytes. Dyes, such as Oil Red $\mathrm{O}$, are cheap, easy to use and widely utilized by laboratories analyzing the adipogenic potential of mesenchymal cells. However, they are not specific to changes in gene transcription. We have developed a gene-specific differentiation assay to analyze when a mesenchymal cell has switched its fate to an adipogenic lineage. Immuno-labelling against fatty acid binding protein-4 (FABP4), a lineage-specific marker of adipogenic differentiation, enabled visualization and quantification of differentiated cells. The ability to quantify adipogenic differentiation potential of mesenchymal cells in a 96 well microplate format has promising implications for a number of applications. Hundreds of clinical trials involve the use of adult mesenchymal stromal cells and it is currently difficult to correlate therapeutic outcomes within and especially between such clinical trials. This simple high-throughput FABP4 assay provides a quantitative assay for assessing the differentiation potential of patient-derived cells and is a robust tool for comparing different isolation and expansion methods. This is particularly important given the increasing recognition of the heterogeneity of the cells being administered to patients in mesenchymal cell products. The assay also has potential utility in high throughput drug screening, particularly in obesity and pre-diabetes research.

\section{Video Link}

The video component of this article can be found at https://www.jove.com/video/57153/

\section{Introduction}

One of the key requirements established by the International Society for Cellular Therapy (ISCT) to define a multipotent mesenchymal stromal cell is that the cells must have the ability to differentiate into the adipogenic, osteogenic and chondrogenic lineages ${ }^{1}$. Conventional methods of measuring differentiation into these three lineages rely on the detection of macromolecular products using chemical dyes ${ }^{1}$. Dyes such as Oil Red O (which stains fat droplets in cells that have undergone adipogenesis), are cheap and easy to use; however they fail to detect the specific changes in gene expression that occur when mesenchymal cells differentiate into each respective lineage ${ }^{2}$. Here, we have developed a differentiation assay which quantifies protein expression for a adipogenic lineage-specific marker, fatty acid binding protein-4 (FABP4) (F, $^{3,5}$. FABP4 was initially found in murine 3T3-L1 adipocytes ${ }^{3}$ and was later discovered to be expressed in human subcutaneous adipose tissue ${ }^{6}$. It is a cytosolic protein, which acts as a chaperone to guide fatty acid uptake by cells and is involved in the process of lipolysis ${ }^{4}$.

The precursor cells used for the differentiation assays were adipose derived mesenchymal stromal cells (ASCs) ${ }^{7,8}$. ASCs share many properties with bone marrow-derived mesenchymal stem cells (BM-MSCs), a major mesenchymal stem cell population in adults ${ }^{8,9}$. ASCs offer several advantages over BM-MSCs in a clinical application, as greater yield of cells can be isolated from more readily accessible tissue sources ${ }^{8,9}$. An isolated cell population needs to meet certain criteria to be defined as ASCs. First, they must show adherence to plastic tissue culture vessels in standard culture conditions ${ }^{1}$. They also must show specific surface antigen expression ${ }^{1}$. Uncultured ASCs are characterized by positive surface antigen expression of CD34, CD73, CD90, low expression of CD105 and negative expression of CD45 and HLA-DR ${ }^{10}$. ASCs purified by culturing 
on plastic for 28 days (adherent purified ASCs) show positive expression of CD73, CD90 and CD105 and negative expression of CD34, CD45 and HLA-DR ${ }^{10}$. Finally, cells must retain the ability to differentiate into various lineages ${ }^{1,7,8}$.

Adipogenic differentiation protocols induce striking upregulation of FABP4 expression amongst other adipocyte lineage genes, so we used immunochemistry to visualize FABP4 protein within cells, and then quantified FABP4 expression at the single cell level using an automated fluorescent high-content screening microscope. This method is advantageous over traditional dyes as it enables highly specific confirmation of adipocyte-lineage differentiation. Such gene specific lineage assays combined with high content screening methods also enable quantification of the proportion of cells within a heterogeneous cell preparation that are capable of differentiation down a particular lineage. In our studies we used the FABP4 assay to confirm loss of adipogenic differentiation potential of freshly isolated ASCs after cell culture.

\section{Protocol}

\section{Preparing ASCs for Differentiation Assays}

1. Prepare tissue culture reagents and handle live cells in a sterile tissue culture hood.

2. Prepare A0 medium: Dulbecco Modified Eagle Medium and Ham's F12 Medium (DMEM/F12), 1x glutamax, 1x penicillin/streptomycin.

3. Prepare complete ASC medium: DMEM/F12, 1x glutamax, 1x penicillin/streptomycin, $10 \%$ fetal bovine serum.

4. Use adherent purified ASCs, expanded in a T75 culture flask. Confirm the purity of ASCs using fluorescence-activated cell sorting (FACS) ${ }^{10}$.

5. Detach cells by removing all medium from the T75 culture flask and adding $2 \mathrm{~mL}$ of cell dissociation enzyme.

6. Leave the culture flask in the incubator $\left(37^{\circ} \mathrm{C}\right.$, humidified, $\left.5 \% \mathrm{CO}_{2}\right)$ for $5-10 \mathrm{~min}$. Take the culture flask out of the incubator and firmly tap the side of flask. Check if the cells have detached from the culture flask using an inverted microscope.

7. Add $13 \mathrm{~mL}$ of $\mathrm{A} 0$ medium to a $\mathrm{T75}$ culture flask.

8. Transfer $15 \mathrm{~mL}$ into a $15 \mathrm{~mL}$ centrifuge tube and centrifuge at $400 \times \mathrm{g}$ for $5 \mathrm{~min}$.

9. Discard the supernatant and re-suspend the cell pellet in $1 \mathrm{~mL}$ of complete ASC medium.

10. Perform a cell count using hemocytometer.

11. Dilute the cell suspension to a concentration of 25,000 cells $\mathrm{mL}^{-1}$ in complete ASC medium.

12. Add $200 \mu \mathrm{L}$ of $\mathrm{A} 0$ medium to all periphery wells of the 96 well plate (flat bottom). This reduces the evaporation rate of the wells containing cells in the center.

13. Transfer $200 \mu \mathrm{L}$ of cell suspension to a microwell plate to achieve a final cell density of $5 \times 10^{3}$ cells per well. Four wells are needed for each treatment group (triplicate technical repeats and a no primary antibody control for immunocytochemistry (ICC)).

14. Leave microwell plate in the incubator $\left(37^{\circ} \mathrm{C}\right.$, humidified, $\left.5 \% \mathrm{CO}_{2}\right)$ until cells reach confluence (3-4 days).

\section{Inducing Differentiation of ASCs}

1. Prepare adipogenic differentiation medium by supplementing complete ASC medium with $1 \mu \mathrm{M}$ dexamethasome, $10 \mu \mathrm{M}$ insulin and $200 \mu \mathrm{M}$ indomethacin. The complete differentiation medium is stable at $4{ }^{\circ} \mathrm{C}$ for 1 month, so only make as required for 1 assay. Store at $4{ }^{\circ} \mathrm{C}$ and when required heat an aliquot to $37^{\circ} \mathrm{C}$ in water bath.

2. After 3-4 days, take microwell plate out of incubator and replace half of the culture medium with adipogenic differentiation medium.

3. Perform a medium change every 2-3 days. NOTE: Optimal adipogenic differentiation requires 14 days of culture in differentiation medium. Differentiated cells are analyzed using traditional dye based stains and gene-specific stains.

\section{Staining for Differentiation - Traditional Dye Based Stains}

1. Prepare $4 \%$ formaldehyde solution by diluting $16 \%$ formaldehyde with PBS. Dilute only the amount needed for experiment and store tightly sealed in a centrifuge tube.

CAUTION: Formaldehyde is a potential carcinogen and can cause irritation to nose, throat and lungs upon inhalation. Perform all procedures relating to formaldehyde in a fume hood.

2. Prepare Oil Red O stock solution by dissolving $300 \mathrm{mg}$ in $100 \mathrm{~mL}$ of isopropanol.

3. Take the microwell plate out of the incubator. The following steps can be performed in a non-sterile environment

4. Remove all medium from wells and fix cells with $4 \%$ formaldehyde for $30 \mathrm{~min}$.

5. During incubation time, prepare Oil Red O working solution. Working solution comprises 6 parts Oil Red O stock with 4 parts distilled water $\left(\mathrm{dH}_{2} \mathrm{O}\right)$. Mix well and let sit at room temperature for $20 \mathrm{~min}$, before filtering using $0.2 \mu \mathrm{m}$ filter unit. Working solution is only stable for $2 \mathrm{~h}$.

6. Remove all formaldehyde from wells by pipetting.

7. Wash cells once with $60 \%$ isopropanol and let the wells dry completely before proceeding.

8. Add $50 \mu \mathrm{L}$ of Oil Red $\mathrm{O}$ working solution to each well and incubate at room temperature for $10 \mathrm{~min}$.

9. Remove Oil Red $\mathrm{O}$ solution and immediately add $\mathrm{dH}_{2} \mathrm{O}$. Wash with $\mathrm{dH}_{2} \mathrm{O} 4$ times before viewing under light microscope.

\section{Staining for Differentiation - Gene Specific Antibodies}

1. Prepare stock solution of Tris buffered saline (TBS) by dissolving $80 \mathrm{~g}$ of $\mathrm{NaCl}, 2 \mathrm{~g}$ of KCl and $30 \mathrm{~g}$ of Tris $\mathrm{Base}$ to $1 \mathrm{~L}$ of $\mathrm{dH} \mathrm{H}_{2} \mathrm{O}(\mathrm{pH} 8)$. Prepare working solution by diluting $1: 10$ using $\mathrm{dH}_{2} \mathrm{O}$. Store at room temperature.

2. Prepare immunobuffer (IB) (1\% fetal bovine serum (FBS) in TBS). Label the $15 \mathrm{~mL}$ centrifuge tube with date and store at $4{ }^{\circ} \mathrm{C}$. IB can be used for 1 week from the date made. 
3. Prepare $0.25 \%$ casein blocker by dissolving $0.5 \mathrm{~g}$ of casein and $0.195 \mathrm{~g}$ of sodium azide in $200 \mathrm{~mL}$ of phosphate buffered saline (PBS). Completely dissolve the ingredients by stirring overnight. Aliquot into $15 \mathrm{~mL}$ centrifuge tubes and store in a $-20^{\circ} \mathrm{C}$ freezer. Thawed casein solutions can be kept at $4{ }^{\circ} \mathrm{C}$ for 1 month.

4. Prepare DAPI solution by diluting stock DAPI 1:200 in TBS. Store at $4{ }^{\circ} \mathrm{C}$, protected from light.

5. Prepare stock thimerosal solution $\left(10 \mathrm{mg} \mathrm{mL}^{-1}\right)$ by dissolving thimerosal in sterile PBS. Store at $4{ }^{\circ} \mathrm{C}$ and dilute appropriate amounts to 0.4 $\mathrm{mg} \mathrm{mL}^{-1}$ for use.

6. Fix and permeabilize cells with ice-cold methanol (96 well plate) for $5 \mathrm{~min}$. Wash cells once with $200 \mu \mathrm{L}$ of TBS.

7. Block with $50 \mu \mathrm{L}$ of $0.25 \%$ casein at room temperature for $10 \mathrm{~min}$ and wash once with TBS.

8. Add $50 \mu \mathrm{L}$ of primary antibodies diluted in IB (see Table 1 for antibody details) and incubate with lids closed for $1 \mathrm{~h}$.

9. Wash once with $200 \mu \mathrm{L}$ of TBS, and then 3 times with TBS for 5 min with gentle rocking.

10. Add $50 \mu \mathrm{L}$ of secondary antibodies diluted in IB (see Table 2 for antibody details) with 1:2000 DAPI in each, incubate with lids closed for $1 \mathrm{~h}$. Cover the plate with foil to prevent photo-bleaching.

11. Wash once with TBS, and then twice with TBS for 15 min with gentle rocking.

12. Remove all TBS and add $200 \mu \mathrm{L}$ of thimerosal solution.

13. Store plate at $4^{\circ} \mathrm{C}$, protected from light until imaging.

\section{Imaging Cells using a High-Throughput Microscope}

1. Analyze cells stained with gene specific antibodies and fluorophore conjugated secondary antibodies (immunofluorescence) using a high content screening microscope controlled with accompanying software.

2. Load the microwell plate into the microscope stage. On the plate acquisition control, select image at medium range magnification (10X objective lens). Make sure images are taken from the center of each well, with $50 \mu \mathrm{m}$ spacing in between each site to ensure that one cell does not appear in two adjacent fields.

3. Select the wells to image.

4. Select the appropriate channel combinations, exposure time and z-offset parameters. Refer to Table $\mathbf{3}$ for more detailed information of each filters (Supplementary Table 2).

5. Acquire the assay using the acquisition wizard (images are automatically stored to Database server).

6. Use alternative channel combinations to acquire plates stained with Oil Red O. (Supplementary Table 3).

\section{Analyze Acquired Image}

Note: Remote access to the database server enables quick and easy evaluation of the images acquired and use of the image analysis software.

1. Open Cell Scoring module on the analysis software.

2. Detect cell nuclei using DAPI channel (nuclear marker) and segment nuclei of interest with user defined parameters for size and signal intensity above background.

3. Detect FABP4 signal using FITC channel. Segment differentiated cells with user defined parameters for size and signal intensity above background to select all FABP4 positive regions in each image.

4. Open Transfluor module on the analysis software to analyze plates stained with Oil Red O.

5. Specify the size and intensity of Oil Red O stained regions as 'pits'.

\section{Representative Results}

In this study, the adipogenic differentiation potential of ASCs with 3 different methods were measured. Immunofluorescent labelling of FABP4, showed that this marker localised to the cytoplasm of differentiated cells, and the labelling overlapped with DAPI-labelled nuclei (Figure 1A). Automated image analysis revealed a 24.6 fold increase in the \% of FABP4 positive cells in the differentiated group in early passage cells compared to 6.9 fold increase in late passage cells (Figure 1B). Oil Red O staining was localized to fat droplets dispersed throughout the cytosol of differentiated cells, and the labelling rarely overlapped with DAPI-labelled nuclei; however from visual inspection, there are fewer cell nuclei associated with Oil Red $\mathrm{O}$ fat droplets in the late passage cells compared to early passage cells (Figure 2A). Automated image analysis revealed an 11.1 fold increase in the area of Oil Red O staining per cell in early passage cells and a 53.9 fold increase in staining area per cell in late passage cells (Figure 2B). Imaging of both stains was performed, and then quantified using Cell Scoring (FABP4) or Transfluor (Oil Red O) module in the software (Supplementary Figure 1, Supplementary Table 2-5). The upregulation of FABP4 expression was confirmed by Western blot analysis (Figure 3, Supplementary Figure 5). All 3 analysis methods revealed that early passage ASCs have higher adipogenic differentiation potential than late passage cells. The adipogenic differentiation did not affect total cell number per well (Supplementary Figure 2, 3). However, the nuclei size of FABP4-positive differentiated cells was significantly smaller than control cells for late passage ASCs only (Supplementary Figure 3).

We demonstrated that as cells were expanded in culture, or passaged, the percentage of cells within the culture capable of adipogenic differentiation decreased, consistent with previously published data ${ }^{11}$. It is important to note that factors such as age, body mass index or previous medical history ${ }^{12}$ could not be controlled for in this study and likely contributed to the variability observed between different donor samples (Supplementary Table 1). 


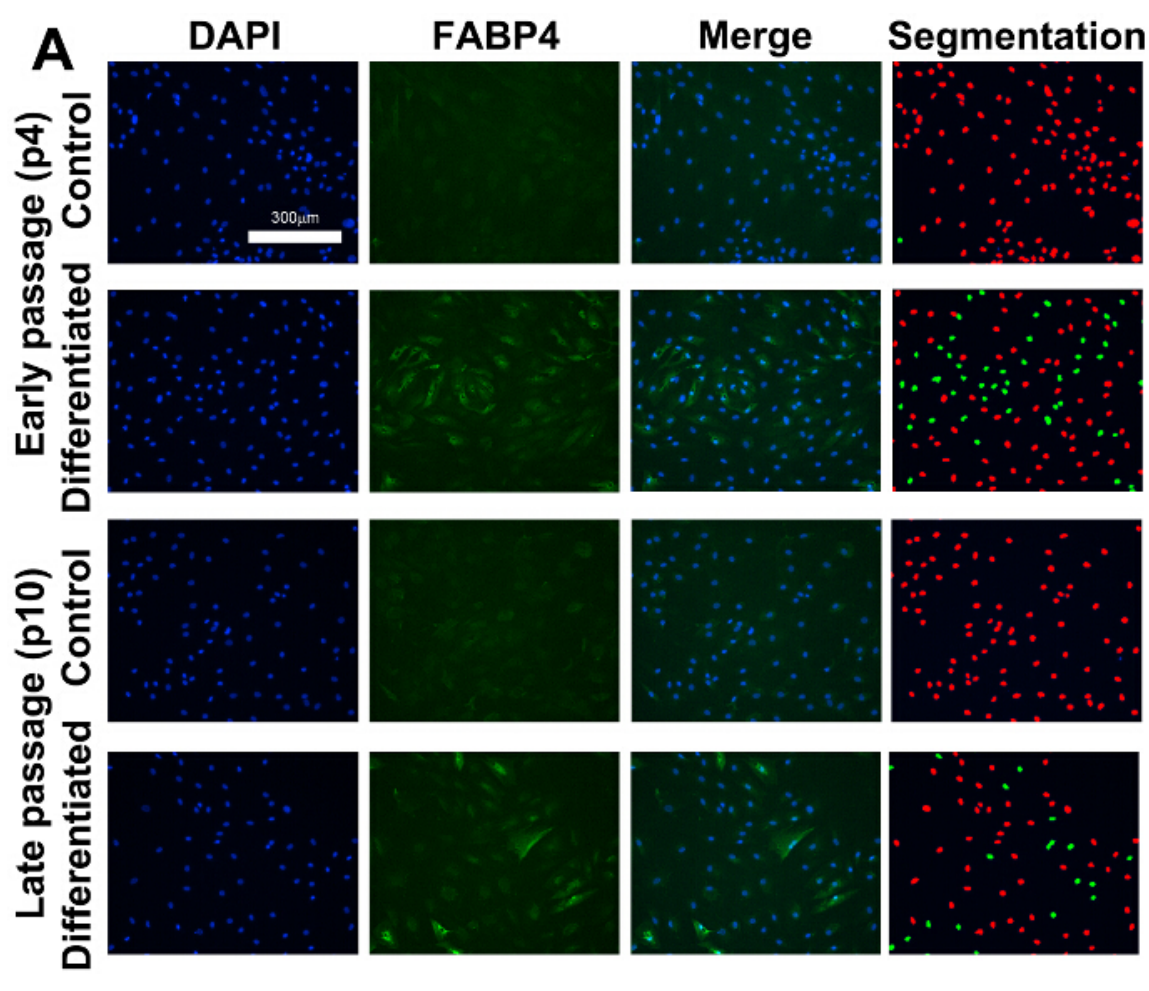

B

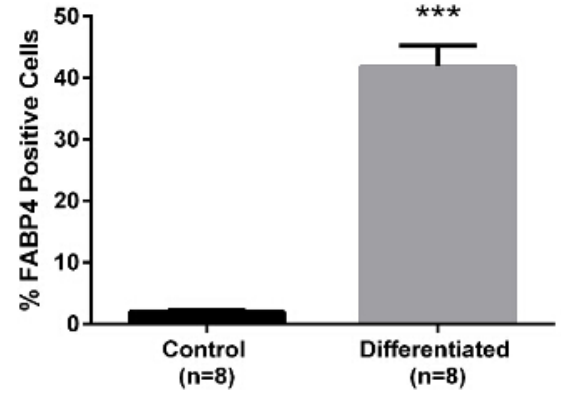

Early passage (p4) Adipogenic differentiation

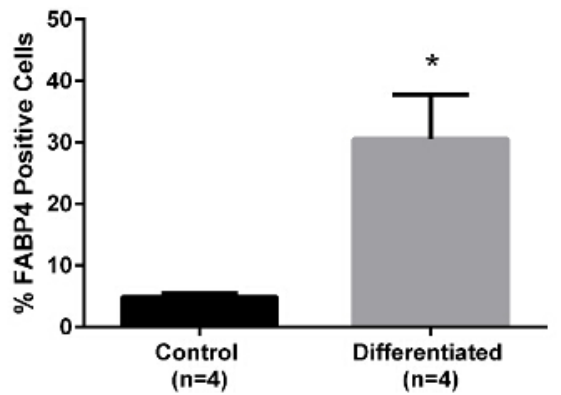

Late passage (p10) Adipogenic differentiation

Figure 1: FABP4 immunolabelling shows that early passage cells have greater differentiation potential than later passage cells. A) Representative images of early and late passage, control and differentiated cells labelled with DAPI (nuclear stain) and FABP4 are shown alongside merge and segmentation images. The segmentation images display differentiated cells with a green overlay and undifferentiated cells with a red overlay. B) A significant increase in FABP4 expressing cells was observed with adipogenic differentiation in early and late passage cells, however, early passage cells demonstrated significantly greater increase in differentiation than late passage cells (Mann Whitney test * denotes $\mathrm{P}<0.05$ and ${ }^{* * *}$ denotes $\left.\mathrm{P}<0.001\right)$. The data shown is the average across early passage $(\mathrm{p} 4 ; \mathrm{n}=8)$ or late passage $(p 10 ; n=4)$ donor samples, \pm SEM. Please click here to view a larger version of this figure. 

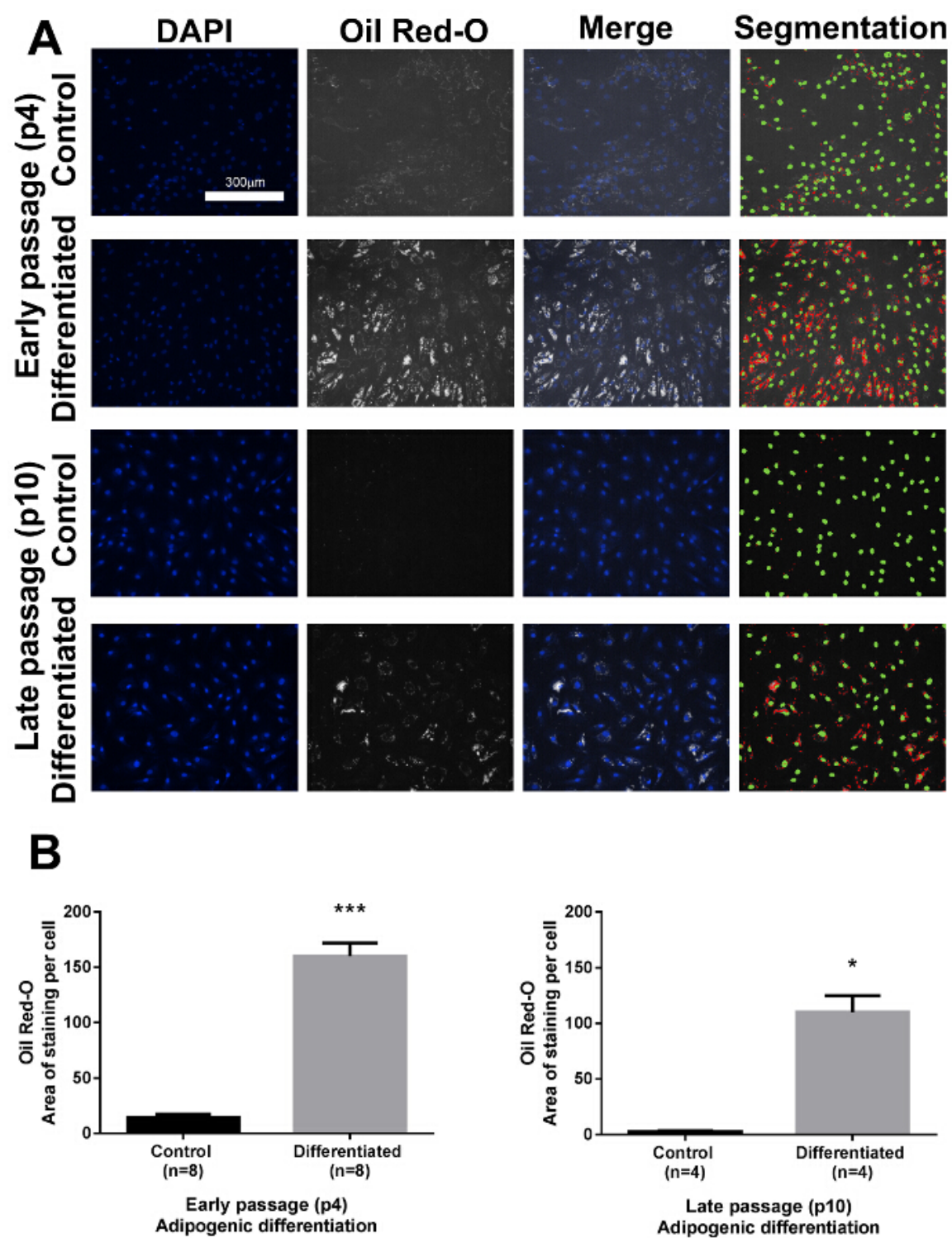

Figure 2: Oil Red $O$ staining shows that early passage cells have greater differentiation potential than later passage cells. A) The representative images of DAPI (nuclear stain) and Oil Red O (grey, lipid droplet stain) are shown alongside merge and segmentation images. The segmentation images depict all nuclei with green overlay and Oil Red O stained lipid droplets with a red overlay. B) A significant increase in Oil Red O staining area was observed with adipogenic differentiation. Early passage cells showed greater area of Oil Red O stain per cell compared to late passage cells. The area of Oil Red O staining per cell $\left(\mu \mathrm{m}^{2}\right)$ is used here as a measure of adipogenic differentiation potential. The data shown is the average across early passage $(p 4 ; n=8)$ or late passage $(p 10 ; n=4)$ donor samples, \pm SEM (Mann Whitney test * denotes $\mathrm{P}=<0.05$ and ${ }^{* * *}$ denotes $\left.\mathrm{P}=<0.001\right)$. Please click here to view a larger version of this figure. 
Early passage (p4)

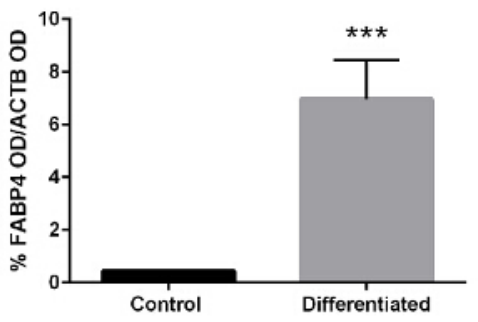

Late passage $(\mathrm{p} 10)$

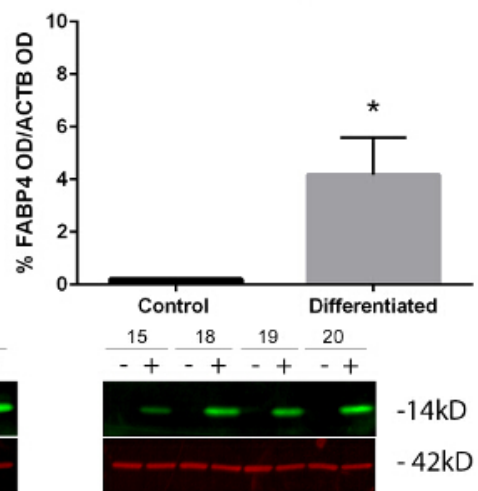

Figure 3: Western blot analysis of FABP4 protein expression level of differentiated early and late passage ASCs. Semi-quantitative analysis of the optical density of FABP4 bands as a ratio of total protein loading control, beta actin (ACTB), showed that FABP4 immunolabelling was significantly increased in early passage and to a lesser degree late passage differentiated cells. The data shown is the average across early passage $(p 4 ; n=8)$ or late passage $(p 10 ; n=4)$ donor samples, \pm SEM (Mann Whitney test * denotes $P=<0.05$ and ${ }^{* * *}$ denotes $\left.P=<0.001\right)$. Please click here to view a larger version of this figure.

\begin{tabular}{|l|l|l|l|}
\hline Target antigen & Isotype (clone) & Species & Dilution \\
\hline FABP4 & Polyclonal & Rabbit & $1: 100$ \\
\hline
\end{tabular}

Table 1: Primary antibody

\begin{tabular}{|l|l|l|l|}
\hline Targeting antibody & Fluorophore label & Species & Dilution \\
\hline Rabbit IgG & Alexa fluoro 488 & Goat & $1: 200$ \\
\hline
\end{tabular}

Table 2: Secondary antibody

Supplementary Figure 1: Overview of the image analysis virtual environment. The stained cells were imaged using an automated, highthroughput fluorescent microscope to avoid any source of bias. Images acquired using ImageXpress Micro XLS, were saved directly to the MDCStore Data Manager database, and made available for viewing through virtual desktop connections, which users use to optimize and test their specific analysis parameters. Images were analyzed with a dedicated 'autorun' instance which enables multiple different users to send 'jobs' to the autorun queue. Users can retrieve their data via the virtual instance once their 'jobs' are completed. Please click here to download this figure.

Supplementary Figure 2: Comparison of total cell count per well of control and differentiated wells for early and late passage ASCs, using FABP4 stained plate. The data shown is the average across early passage $(p 4 ; n=8)$ or late passage $(p 10 ; n=4)$ donor samples, \pm SEM. There was no statistically significant difference between control and differentiated cells for both early and late passage ASCs. There were more cells per well for early passage cells compared to late passage cells. Please click here to download this figure.

Supplementary Figure 3: Comparison of total cell count per well of control and differentiated wells for early and late passage ASCs, using Oil Red $O$ stained plate. The data shown is the average across early passage $(p 4 ; n=8)$ or late passage $(p 10 ; n=4)$ donor samples, \pm SEM. There was no statistically significant difference between control and differentiated cells for both early and late passage ASCs. Please click here to download this figure.

Supplementary Figure 4: At later passages, differentiated cells that were FABP4 positive had significantly smaller nuclei area than cells in control wells. There was no statistically significant difference in nuclei area between control and differentiated cells in early passage. The data shown is the average across early passage $(p 4 ; n=8)$ or late passage $(p 10 ; n=4)$ donor samples, \pm SEM. (Unpaired t test. ${ }^{* \star *}$ denotes $P=<0.001)$. Mean \pm SEM are shown.) Please click here to download this figure.

Supplementary Figure 5: Images of Western blot gels. Red channel depicts beta-actin. Green channel depicts FABP4 immunolabelling. Please click here to download this figure.

Supplementary Table 1: Clinicopathological information of donors Please click here to download this table.

Supplementary Table 2: Imaging settings (FABP4) Please click here to download this table.

Supplementary Table 3: Imaging settings (Oil Red O) Please click here to download this table.

Supplementary Table 4: Table of analysis parameters used (FABP4). Cell Scoring module. Please click here to download this table.

Supplementary Table 5: Table of analysis parameters used (Oil Red O). Trans Fluor module. Please click here to download this table. 


\section{Discussion}

This paper demonstrates the utility and advantages of FABP4 immunolabelling to accurately detect and quantify mature adipocytes derived from mesenchymal stromal cells. FABP4 is able to detect changes in expression of a lineage specific protein ${ }^{3,4,5}$ in mature adipocytes,

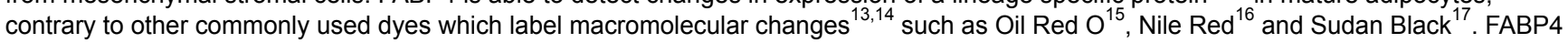
immunolabelling allows for visualization and analysis of changes in cellular morphology. This is a distinctive advantage over previously described methods using quantitative reverse transcription PCR (RT-qPCR) that analyzes changes at the transcript level without any visualisation ${ }^{5,18,19,20}$, or flow cytometry that requires cells to be in suspension ${ }^{20}$, or Western blotting that requires cells to be lysed to isolate protein ${ }^{19,20}$. FABP4 immunolabelling is stable in fixed cells, does not leak out in solution and being a cytosolic protein when labelled in an adherent monolayer of cells, will overlap with the nucleus allowing for easy visualisation and added accuracy in the automated analysis.

High-content screening is advantageous because it is fast, accurate, reproducible and objective. It provides a platform for cost effective use of reagents and researcher time, since image acquisition and analysis require minimal manual manipulation and rely on the automatic processing of the instrument. Several factors were identified as critical to the accuracy and reproducibility of the high-content screening assays ${ }^{31}$. The quantification of antigen expression relies on image quality. For successful image analysis, images from each channel per well must be in focus and fall within an optimal dynamic range for gray values (Auto Expose settings are ideal). Out of focus or saturated images lead to erroneous results.

Some potential limitations of the methods described in this article include the following. The requirement for specialised imaging equipment and analysis software. While outside the scope of this article, alternative open source software options (for example, Image ${ }^{32,33}$, Fiji ${ }^{34}$, CellProfiler ${ }^{32,35}$ ) can be used to perform similar image segmentation tasks; however they do require the skills to either download and tailor macros available online or write macros/commands for their specific analysis pipelines. Inherent to all automated imaging analysis pipelines is a level of background noise. This can largely be attributed to debris, poor image quality or analysis error, emphasizing the need for careful sample preparation and careful set-up of the imaging and analysis platform. The FABP4 label in mice has been found to detect undifferentiated progenitors $^{30}$; while the controls in this study (which consist of undifferentiated cells) are devoid of specific FABP4 staining. This under-scores the necessity of checking FABP4 expression in undifferentiated progenitors in each biological context where the assay is employed.

In order to draw valid comparisons between the FABP4 labelling and Oil Red O staining, the output measurements from image analysis needed to be biologically relevant. Consequently, the measurement, '\% positive cells' was used for FABP4, and 'area of staining per cell' was used for Oil Red O. It is noteworthy that the measure '\% positive cells' was not used for Oil Red O labelled cells in our study because it was not possible to attribute the labelled fat droplets accurately to a given nucleus; the fat droplets varied considerably in size, number and distribution across the cell body and rarely overlapped with the nucleus. Oil Red O staining variability exists between cells derived from different tissue sources; while the $\%$ positive cells measure was not appropriate for the current study, another group has used it successfully to quantify adipocytes derived from human gland stem cells ${ }^{22}$ where the Oil Red O staining appeared as one large fat droplet which spanned the cytoplasm and overlapped with nuclei and enabling data to be presented as $\%$ positive cells.

In contrast, the morphology of FABP4 immunolabelling is consistent in adipocytes derived from a range of different tissue sources ${ }^{23,24,25}$. The ability to quantify the proportion of cells within a culture capable of differentiation has a number of applications. Adult mesenchymal stromal cells hold significant promise for a diverse range of therapeutic uses. A significant issue that has arisen with clinical translation is the inherent variability in the capability of these cells between patients ${ }^{26}$, between sites of isolation ${ }^{27,28}$, and between methods for isolation ${ }^{12}$ and expansion of cell numbers ${ }^{12}$ for therapeutic use. It has been shown previously that cultures of adherent stromal cells are frequently heterogeneous, with some cells capable of differentiation and some not ${ }^{12,17}$ as our FABP4 assay demonstrates for ASC cultures. Assays like this, combined with enrichment techniques, will help identify sub-populations within heterogeneous cultures capable of lineage-specific differentiation. Having a standardized, quantifiable assay such as this FABP4 assay provides a simple method for comparison between all of these variables, and the potential to evaluate therapeutic outcomes within and between clinical trials.

The quantification of FABP4 in a semi-automated fashion enables objectivity and reproducibility in analysis across many donor cases and samples. Furthermore as the label is cytoplasmic, it can potentially be used to analyze hypertrophy (enlargement of adipocyte size) in addition to hyperplasia (increase in adipocyte number), a distinction that is of considerable importance in obesity research, where hypertrophy is strongly associated with adipose dysfunction in obese individuals ${ }^{21}$. The obesity epidemic has spurred a significant amount of interest in identifying drugs capable of controlling lipid storage. This FABP4 assay also provides a simple readout for screening thousands of compounds for their ability to inhibit lipid accumulation.

\section{Disclosures}

The authors declare no conflicts of interest.

\section{Acknowledgements}

We are grateful to the donors of the adipose tissues and the research professionals who collect and provide this resource to us for research use.

We would like to acknowledge funding support by Allergan. We are also grateful to the University of Auckland, Faculty of Medical and Health

Sciences Performance Based Research Fund for funding the cost of videoing and editing for submission of this article. 


\section{References}

1. Dominici, M. et al. Minimal criteria for defining multipotent mesenchymal stromal cells. The International Society for Cellular Therapy position statement. Cytotherapy. 8 (4), 315-317 (2006).

2. Proescher, F. Oil Red O Pyridin, A Rapid Fat Stain. Biotech Histochem. 2 (2), 60-61 (1927)

3. Matarese, V., Bernlohr, D. a. Purification of murine adipocyte lipid-binding protein. Characterization as a fatty acid- and retinoic acid-binding protein. J Biol Chem. 263 (28), 14544-14551 (1988).

4. Lafontan, M., Langin, D. Lipolysis and lipid mobilization in human adipose tissue. Prog Lipid Res. 48 (5), $275-297$ (2009).

5. Sekiya, I., Larson, B.L., Vuoristo, J.T., Cui, J.C., Prockop, D.J. Adipogenic Differentiation of Human Adult Stem Cells From Bone Marrow Stroma (MSCs). J Bone Min Res. 19 (2), 256-264 (2004).

6. Baxa, C. et al. Human adipocyte lipid-binding protein: purification of the protein and cloning of its complementary DNA. Biochemistry. 28 (22), 8683-8690 (1989).

7. Bourin, P. et al. Stromal cells from the adipose tissue-derived stromal vascular fraction and culture expanded adipose tissue-derived stromal/stem cells: A joint statement of the International Federation for Adipose Therapeutics and Science (IFATS) and the International So. Cytotherapy. 15 (6), 641-648 (2013).

8. Zuk, P. et al. Multilineage cells from human adipose tissue: implications for cell-based therapies. Tissue Eng. 7 (2), $211-228$ (2001).

9. Orbay, H., Tobita, M., Mizuno, H. Mesenchymal stem cells isolated from adipose and other tissues: Basic biological properties and clinical applications. Stem Cells Int. 2012 (2012).

10. Feisst, V., Brooks, A.E.S., Chen, C.-J.J., Dunbar, P.R. Characterization of mesenchymal progenitor cell populations directly derived from human dermis. Stem Cells Dev. 23 (6), 631-42 (2014).

11. Mitterberger, M.C., Lechner, S., Mattesich, M., Zwerschke, W. Adipogenic differentiation is impaired in replicative senescent human subcutaneous adipose-derived stromal/progenitor cells. Journals Gerontol - Ser A Biol Sci Med Sci. 69 (1), 13-24 (2014).

12. Baer, P.C., Geiger, H. Adipose-derived mesenchymal stromal/stem cells: Tissue localization, characterization, and heterogeneity. Stem Cells Int. 2012 (2012).

13. Xu, S. et al. Evaluation of foam cell formation in cultured macrophages: An improved method with Oil Red O staining and Dil-oxLDL uptake. Cytotechnology. 62 (5), 473-481 (2010).

14. Hopkins, P.M. et al. Oil red O stain of alveolar macrophages is an effective screening test for gastroesophageal reflux disease in lung transplant recipients. J Hear Lung Transplant. 29 (8), 859-864 (2010).

15. Dragunow, M., Cameron, R., Narayan, P., O'Carroll, S. Image-based high-throughput quantification of cellular fat accumulation. $J$ Biomol Screen. 12 (7) (2007)

16. Greenspan, P., Mayer, E.P., Fowler, S.D. Nile red: a selective fluorescent stain for intracellular lipid droplets. J Cell Biol. 100 (3), 965-973 (1985).

17. Muraglia, a, Cancedda, R., Quarto, R. Clonal mesenchymal progenitors from human bone marrow differentiate in vitro according to a hierarchical model. J Cell Sci. 113 ( Pt 7, 1161-1166 (2000).

18. Neubauer, M. et al. Basic fibroblast growth factor enhances PPARgamma ligand-induced adipogenesis of mesenchymal stem cells. FEBS Lett. 577 (1-2), 277-283 (2004).

19. Janderova, L., McNeil, M., Murrell, A.N., Mynatt, R.L., Smith, S.R. Human mesenchymal stem cells as an in vitro model for human adipogenesis. Obes Res. 11 (1), 65-74 (2003).

20. Aldridge, A., Kouroupis, D., Churchman, S., English, A., Ingham, E., Jones, E. Assay validation for the assessment of adipogenesis of multipotential stromal cells--a direct comparison of four different methods. Cytotherapy. 15 (1), 89-101 (2013).

21. Wang, Q.A., Tao, C., Gupta, R.K., Scherer, P.E. Tracking adipogenesis during white adipose tissue development, expansion and regeneration. Nat Med. 19 (10), 1338-1344 (2013).

22. Gorjup, E., Peter, L., Wien, S., von Briesen, H., Schmitt, D. Automated microscopic quantification of adipogenic differentiation of human gland stem cells. Ann Anat. 191 (1), 13-22 (2009).

23. Wojciechowicz, K., Gledhill, K., Ambler, C.A., Manning, C.B., Jahoda, C.A.B. Development of the mouse dermal adipose layer occurs independently of subcutaneous adipose tissue and is marked by restricted early expression of FABP4. PLoS One. 8 (3), e59811 (2013).

24. Sandhu, M.A., Jurek, S., Trappe, S., Kolisek, M., Sponder, G., Aschenbach, J.R. Influence of Bovine Serum Lipids and Fetal Bovine Serum on the Expression of Cell Surface Markers in Cultured Bovine Preadipocytes. Cells Tissues Organs. 204 (1), 13-24 (2017).

25. LaRosa, P.C. et al. Trans-10, cis-12 conjugated linoleic acid activates the integrated stress response pathway in adipocytes. Physiol Genomics. 31 (3), 544-553 (2007).

26. Sen, A. et al. Adipogenic potential of human adipose derived stromal cells from multiple donors is heterogeneous. J Cell Biochem. 81 (2), 312-9 (2001).

27. Zuk, P.A. et al. Human Adipose Tissue Is a Source of Multipotent Stem Cells. Mol Biol Cell. 13 (12), $4279-4295$ (2002).

28. Russo, V., Yu, C., Belliveau, P., Hamilton, A., Flynn, L.E. Comparison of Human Adipose-Derived Stem Cells Isolated from Subcutaneous, Omental, and Intrathoracic Adipose Tissue Depots for Regenerative Applications. Stem Cells Transl Med. 3 (2), 206-217 (2014).

29. Tilg, H., Moschen, A.R. Adipocytokines: mediators linking adipose tissue, inflammation and immunity. Nat Rev Immunol. 6 (10), $772-783$ (2006).

30. Shan, T., Liu, W., Kuang, S. Fatty acid binding protein 4 expression marks a population of adipocyte progenitors in white and brown adipose tissues. FASEB J Off Publ Fed Am Soc Exp Biol. 27 (1), 277-287 (2013).

31. Narayan, P.J., Dragunow, M. High content analysis of histone acetylation in human cells and tissues. J Neurosci Methods. 193 (1) (2010).

32. Buchser, W. et al. Assay Development Guidelines for Image-Based High Content Screening, High Content Analysis and High Content Imaging. Assay Guid Man. 1-80 (2004).

33. Abràmofff, M.D., Magalhães, P.J., Ram, S.J. Image processing with ImageJ Part II. Biophotonics Int. 11 (7), $36-43$ (2005).

34. Schindelin, J. et al. Fiji: an open-source platform for biological-image analysis. Nat Methods. 9 (7), 676-682 (2012).

35. Carpenter, A.E. et al. CellProfiler: image analysis software for identifying and quantifying cell phenotypes. Genome Biol. 7 (10), R100 (2006). 